\title{
Granulomatous Lymphocytic Interstitial Lung Disease (GL-ILD): CT Imaging Features and Clinical Findings to Expedite a Timely Diagnosis
}

\section{Avanthika Wynn ( $\sim$ a.t.wynn@emory.edu )}

Emory University Hospital

\section{Eugene A. Berkowitz}

Emory University Hospital

Brent P. Little

Massachusetts General Hospital

Srihari Veeraraghavan

Emory University Hospital

\section{Frank Schneider}

Emory University Hospital

\section{Marissa Shams}

Emory University Hospital

Frederic Bertino

Emory University Hospital

\section{Research Article}

Keywords: diagnosis , Granulomatous Lymphocytic-Interstitial Lung Disease (GL-ILD), histologic features

Posted Date: March 1st, 2021

DOl: https://doi.org/10.21203/rs.3.rs-236562/v1

License: (c) (1) This work is licensed under a Creative Commons Attribution 4.0 International License. Read Full License 


\section{Abstract}

Background: Establishing a diagnosis of Granulomatous Lymphocytic-Interstitial Lung Disease (GL-ILD) is difficult due to the overlapping CT imaging and histologic features, and are often confused with infection, sarcoidosis and/or follicular bronchiolitis.

Research Question: Identify specific CT imaging features and clinical characteristics to suggest a confident diagnosis of Granulomatous Lymphocytic-Interstitial Lung Disease (GL-ILD).

Study Design and Methods: IRB-approved retrospective case series study involving the review of the electronic medical record and CT chest imaging of eight patients with GL-ILD.

Results: Bronchocentric airway-centered lower lobe predominant (part solid nodules more common than ground glass or solid nodules) ranging from 5 to $10 \mathrm{~mm}$ in size were found consistently in a majority of the eight patients with GL-ILD.

Interpretation: In combination with the above pulmonary nodular pattern, mediastinal and hilar lymphadenopathy in conjunction, either splenomegaly or splenectomy and/or abdominal lymphadenopathy are excellent CT imaging features to prospectively suggest the diagnosis of GL-ILD in a patient with CVID.

\section{Background}

Granulomatous lymphocytic interstitial lung disease (GL-ILD) is an interstitial lung disease (ILD) that occurs in patients with common variable immunodeficiency (CVID) and is associated with a lymphocytic infiltrate and/or granulomatous inflammation in the lung, and diagnosed when other possible conditions have been excluded [1]. The non-infectious pulmonary complications of CVID include organizing pneumonia, follicular bronchiolitis, GL-ILD, malignancy (lymphoma), obstructive lung disease secondary to bronchiolitis obliterans (BO), bronchiectasis/bronchiolectasis and pulmonary nodules [2, 3]. It is challenging to distinguish the infectious pulmonary manifestations and their associated sequela from noninfectious inflammatory processes as a result of overlapping clinical, radiological and pathological features.

A confident diagnosis of GL-ILD often requires a histological evaluation [4] combined with cultures and special stains to exclude infection $[4,5]$. However, surgical lung biopsies in patients with CVID can be associated with an increased risk of decompensation, infection and surgical complications [6]. It is therefore crucial to recognize and suggest GL-ILD in CVID patients with the appropriate CT imaging pattern to allow for early diagnosis and treatment. GL-ILD can be confused with other common and uncommon diseases like sarcoidosis and/or (follicular) bronchiolitis both histologically and on CT chest imaging [5-8]. Characterization of CT imaging features that allow the radiologist to suggest a confident early diagnosis can reduce this diagnostic dilemma. Recognizing and defining the clinicopathological 
features, as well as the radiological features, of this condition will help improve early identification, confident diagnosis and treatment of this multi-systemic disease [9].

Presence of solid nodules $<3 \mathrm{~cm}$, semisolid nodules, pure ground-glass opacities, enlarged thoracic (hilar and/or mediastinal) lymph nodes, and splenomegaly have been suggested as typical in GL-ILD but none were consistently rated as necessary to make the diagnosis $[1,10]$. There is a need for better defining the radiological features to avoid the risk of complications from surgical biopsies in this high-risk population. To date, there is no clear consensus on the CT imaging features or their distribution for GL-ILD. The purpose of this retrospective case series was to evaluate our cohort of eight patients with CVID that developed a GL-ILD pattern on CT in conjunction with clinical and/or pathologic diagnosis of GL-ILD to establish specific CT imaging features to allow the radiologist to suggest a presumptive diagnosis of GLILD, possibly obviating a potential surgical lung biopsy.

\section{Methods}

\section{Study population -}

Approval was obtained to review the electronic medical record for retrospective case series by the institutional review board of Emory University and was performed in accordance with the Code of Ethics of the World Medical Association (Declaration of Helsinki). The electronic medical record was used to search for patients with a diagnosis of CVID made by an immunologist from 1995 to 2018 and a confirmed diagnosis of GL-ILD by a multidisciplinary discussion (MDD) approach involving the interstitial lung disease (ILD) team comprised of ILD pulmonologists, immunologist, thoracic radiologist and pathologists (Table 1). The electronic medical record was reviewed of those with CVID and a cohort of patients with a confirmed diagnosis of CVID and chest CT imaging from 1995 to 2018 (Table 1). All pertinent clinical information was retrieved from the electronic medical record, including demographics, pulse oximetry readings, symptoms, pulmonary function test (PFTs) data, histopathology and laboratory results.

\section{CT evaluation -}

The CT scans used for scoring was the most recent CT chest; however, previous CT scans were referred to when needed for comparison. The presence of an active infection was excluded by chart review, cultures and special stains. CT chest imaging was performed on a variety of scanners from several different manufacturers within our institution: GE Lightspeed VCT spiral acquisition, 120 to 140 KVP, slice thickness 1.25 to $2.5 \mathrm{~mm}$, DFOV of 30 to $33 \mathrm{~cm}$, ASIR 30; Seimens Somaton Definition AS spiral acquisition, $120 \mathrm{KVP}$, slice thickness of $1 \mathrm{~mm}$, DFOV of $33 \mathrm{~cm}$; Seimens Somaton Force spiral acquisition, KVP 110, DFOV of $34 \mathrm{~cm}$, slice thickness of $2 \mathrm{~mm}$; Seimens Somaton Flash spiral acquisition, KVP 100, DFOV of $42 \mathrm{~cm}$, slice thickness of $2 \mathrm{~mm}$. 


\section{Method -}

Two thoracic radiologists (EB with 12 years and $\mathrm{BL}$ with 10 years of experience in thoracic radiology) independently reviewed CT scans and scored according to a scoring sheet agreed upon by the study team prior to reviewing the CT scans. Disagreements were resolved by consensus. Data was collected on the following parameters: the presence, absence, attenuation/density, size and distribution of pulmonary nodules; the presence, absence and distribution of bronchiectasis; the presence, absence, axial distribution, size of ground glass opacities; presence or absence of bronchial wall thickening, air trapping/mosaic attenuation, pleural effusions, consolidations, hepatomegaly, splenomegaly, abdominal lymphadenopathy and hilar/mediastinal lymphadenopathy.

\section{Results}

Our case series had eight patients, all non-Hispanic white 5 females and 3 males, ranging in age from 30 to 66; five of the eight patients had immune thrombocytopenic purpura (ITP) (Table 1). Five out of the eight patients had symptoms at presentation. Only two of the patients were ex-smokers (case 3 - 5 pack years, case 8 - 20 pack years). Case 3 and 7 had hypoxia requiring oxygen therapy. Case 3 was on treatment for a B cell lymphoma diagnosed by subcarinal lymph node biopsy and flow cytometry (Figure $1 \mathrm{E}$ and $1 \mathrm{G}$ ). Three patients were on rituximab for treatment of GL-ILD. Seven of the eight patients had pulmonary function testing (PFT) and five patients have had full PFT with lung volume testing. The most predominant PFT pattern was a reduced diffusion capacity of carbon monoxide (DLCO) seen in four patients. Restrictive lung functions were seen in three patients. Airflow limitation/obstructive pattern was not seen in any of the patients. (Table 1)

Table 2 summarizes imaging data with regard to nodule distribution and density, and the presence or absence of bronchiectasis, reticulation, splenomegaly, and lymphadenopathy. The distribution of nodules is bronchocentric with a lower lobe predominance (Table 2). All the lobes had more than 10 nodules bilaterally in all 8 patients. The largest nodule size was $\geq 10 \mathrm{~mm}$ in all the lobes. Overall, the most common nodule density was part solid (Figures 1 and 2) but solid and ground glass nodules were present in all except for 1 patient who had predominantly part solid nodules. Bronchiectasis was not a common finding and when present, was very mild, cylindrical and lower lobe predominant. Mild to moderate smooth bronchial/bronchiolar wall thickening was present in all eight patients (Figure 1B). Lower lobe predominant patchy ground glass opacities were seen on the CT chest in seven patients (Figure 2). Three patients had mild lobular geographic areas of mosaic attenuation representing air trapping, such as, patient 4 which demonstrated lower lobe predominant small part solid, solid and ground glass nodules (Figure $3 \mathrm{~A}$ ), representing follicular bronchiolitis and scattered poorly-formed non-necrotizing granulomas (Figure 3B). Lower lobe predominant fine reticulations were present in four of the eight patients. None of the patients had consolidation or pleural effusion. Five patients had splenomegaly (Figure 1F) while the other three patients had splenectomies due to previous splenomegaly; none of the patients had hepatomegaly. Seven patients had abdominal lymphadenopathy. Mediastinal lymphadenopathy (Figure $1 \mathrm{D}$ and $\mathrm{E}$ ) was seen in all eight patients and hilar lymphadenopathy (Figure 1E) in seven patients. Figure 
4 demonstrates the apical to basal gradient of the solid, part-solid and ground glass nodules, most prominent in the lower lobes with intermixed patchy areas of ground glass opacities. An early confident diagnosis allowed treatment to start early with a positive response to therapy as demonstrated in Figure 5 .

\section{Discussion}

CVID is a primary immunodeficiency characterized by decreased IgG two standard deviations below the lower limits of normal and reduced IgA and/or IgM with impaired specific antibody response measured via vaccine titers and variable $T$ cell defects [3], that cause recurrent infections, particularly upper and lower respiratory tract infections [2]. GL-ILD is a non-infectious complication of CVID that manifests various lung findings on $\mathrm{CT}$ imaging that have not been previously well characterized with respect to morphology and distribution. Additionally, there are several histological patterns including granulomatous inflammation, lymphoid hyperplasia/lymphocytic interstitial pneumonia (LIP) and follicular bronchiolitis $[2,9,11]$ that can be confusing to the pathologist. A retrospective series that examined lung pathology in 34 patients with CVID demonstrated benign lymphoid proliferation and granulomas in 23 patients who did not have a clinical and radiological diagnosis of GL-ILD [5]. Over-diagnosing GL-ILD in patients histologically without tight clinical, historical and radiological criteria and multidisciplinary agreement does not provide useful information in managing patients. Patients with CVID may have other pulmonary manifestations such a bronchiectasis, necrotizing granulomas from infection, lymphocytic intestinal pneumonia (LIP) from coexisting autoimmune disease[5, 12], scarring and pneumonia that may overlap with GL-ILD [13]. Some of the CT imaging features associated with GL-ILD are common to sarcoidosis [14], and are thus often misdiagnosed as sarcoidosis, especially histologically [6, 7, 14]. Histology may not be the "gold standard" in diagnosing GL-ILD rather a multidisciplinary ILD conference is the most optimal method to obtain a diagnosis of GL-ILD with the radiologist raising GL-ILD in the differential diagnosis. In centers with multidisciplinary ILD teams, a lung biopsy can be reserved to resolve disagreements and ambiguity.

Although GL-ILD suggests a pulmonary process, it actually encompasses a multi-systemic granulomatous inflammatory disease that may affect the liver, spleen, lymph nodes and any organ/organ system [15]. Due to the lymphocytic dysregulation in GL-ILD, patients are at high risk of lymphoma [16]. Several theories have been proposed to explain the pathogenesis of GL-ILD in CVID, including dysfunctional antigen handling (due to impaired T cell function) and aberrant immune response to viruses (potentially HHV-8). Due to improved life expectancy and reduced rate of infection with immunoglobulin replacement therapy, non-infectious manifestations of CVID such as GL-ILD are becoming more emergent $[6,11]$. GL-ILD treatment requires immunomodulatory therapy (rituximab, azathioprine), as well as high dose immunoglobulin replacement therapy $[13,17]$ to reduce the disease progression and potentially reverse some of the radiological disease [9]. Some patients diagnosed with GL-ILD with normal lung functions and mild disease may not need treatment unless there is radiological and clinical progression [18]. 
The main goal of our case series was to identify predominant clinical and imaging findings in this cohort of GL-ILD patients that can be used to identify patients with CVID who have developed GL-ILD. The lower lobe predominant ground glass opacities (GGOs) (7 out of the 8 patients) were a finding more common in prior CT scans that tend to evolve into solid nodules in the subsequent scans. While longitudinal imaging studies are not available, it can be theorized that these GGOs are nodules in evolution, based on their distribution. There was $98 \%$ agreement between the two independent chest radiologists for the individual radiological findings of bronchocentric nodules with an apical to basal gradient, most numerous in the lower lobes.

When a PFT abnormality was present, the predominant finding was a moderate to severe reduction in the diffusion capacity, two patients who had severe impairment of diffusion capacity had hypoxia (Table 1). Restrictive lung functions are the predominant finding in GL-ILD in keeping with previous studies and case reports $[11,12,18]$; no patient had airflow limitation/ obstructive physiology that would implicate airway involvement[12]. Splenomegaly and ITP were important predictors of GL-ILD in CVID patients that were identified by the case control study by Hartano et al (69\%) [6]. Splenomegaly was also an important clinical finding towards establishing a diagnosis of GL-ILD in the BLS/UKPIN statement [1], five patients in our series demonstrated splenomegaly while the other three patients had a splenectomy (Table 2). Features of ITP were present in five patients. Mild to moderate cylindrical bronchiectasis that was found in six patients is representative of the sequel of recurrent infections in CVID rather than a feature of GLILD.

Limitations of the GL-ILD case series is the small sample size of eight, but representative of the prevalence of this rare disease and the strict case selection by the multidisciplinary ILD team. Additional cohorts in a multicenter approach would be necessary to validate these findings. This study has the restrictions of a retrospective chart review and our data is limited by the availability of test results and documentation in the chart. One of the strengths that stand out in this study is the in-depth explanation of the radiological findings with CT imaging examples from our cohort.

Future studies should focus on multi-center database approach to increase the sample size of this rare disease. HRCT and better defined radiological criteria are replacing the need for biopsy in the field of ILD. GL-ILD has distinct radiological findings that can be useful in centers with multidisciplinary ILD teams.

\section{Interpretation}

Bronchocentric airway-centered lower lobe predominant (part solid nodules more common than ground glass or solid nodules) ranging from 5 to $10 \mathrm{~mm}$ in size were found consistently in a majority of the eight patients confirmed with GL-ILD. In combination with the above pulmonary nodular pattern, mediastinal and hilar lymphadenopathy in conjunction, either splenomegaly or splenectomy and/or abdominal lymphadenopathy are excellent CT imaging features to prospectively suggest the diagnosis of GL-ILD in a patient with CVID. 


\section{Abbreviations}

BO- bronchiolitis obliterans

CVID - common variable immunodeficiency

DLCO - Diffusion capacity of carbon monoxide

GL-ILD - Granulomatous lymphocytic interstitial lung disease

ILD - Interstitial lung disease

ITP - Idiopathic thrombocytopenic purpura

PFT - Pulmonary function test

\section{Declarations}

\section{Ethics approval and consent to participate:}

The Emory University Institutional Review Board (IRB) approval for retrospective case series was obtained. Informed consent waived for retrospective data review process.

\section{Consent for publication:}

Not applicable

\section{Availability of data and materials:}

All data generated or analyzed during this study are included in this published article

\section{Competing interests:}

No conflicts of interest for AW, BL, MS, SV, FB, FS or EB

\section{Funding :}

Not applicable

\section{Meeting abstract:}


An abstract containing a portion of the data with AW as the first author was presented at CHEST Annual Meeting October 6-10, 2018 in San Antonio, Texas. https://journal.chestnet.org/article/S00123692(18)31594-0/abstract

\section{Authors' contributions :}

AW and EB gathered data and prepared the manuscript.

$\mathrm{EB}$ and $\mathrm{BL}$ independently interpreted and reviewed the imaging for the study.

EB and FB prepared radiology figures and captions.

FS interpreted and prepared the pathology. FS prepared the pathology pictures and the captions.

All authors reviewed the manuscript.

\section{Acknowledgements}

Eugene Berkowitz and Brent Little for analyzing imaging.

EB and ATW are guarantors for the data integrity and preparing the manuscript.

EB, FS and FB for contributing to image preparation and figure legends.

SV, MS for contributing with expertise in the field and manuscript review

\section{References}

1. Hurst, J.R., et al., British Lung Foundation/United Kingdom Primary Immunodeficiency Network Consensus Statement on the Definition, Diagnosis, and Management of Granulomatous-Lymphocytic Interstitial Lung Disease in Common Variable Immunodeficiency Disorders. J Allergy Clin Immunol Pract, 2017; 5(4): 938-945.

2. Park, J.H. and A.I. Levinson, Granulomatous-lymphocytic interstitial lung disease (GLILD) in common variable immunodeficiency (CVID). Clin Immunol, 2010; 134(2): 97-103.

3. Resnick, E.S. and C. Cunningham-Rundles, The many faces of the clinical picture of common variable immune deficiency. Curr Opin Allergy Clin Immunol, 2012; 12(6): 595-601.

4. Shah, J.L., et al., Granulomatous-Lymphocytic Interstitial Lung Disease in a Patient With Common Variable Immunodeficiency. Curr Probl Diagn Radiol, 2018; 47(4): 282-284. 
5. Larsen, B.T., et al., GLILD Revisited: Pulmonary Pathology of Common Variable and Selective IgA Immunodeficiency. Am J Surg Pathol, 2020; 44(8):1073-1081.

6. Hartono, S., et al., Predictors of granulomatous lymphocytic interstitial lung disease in common variable immunodeficiency. Ann Allergy Asthma Immunol, 2017; 118(5): 614-620.

7. Verbsky, J.W. and J.M. Routes, Sarcoidosis and common variable immunodeficiency: similarities and differences. Semin Respir Crit Care Med, 2014; 35(3): 330-5.

8. Bouvry, D., et al., Granulomatosis-associated common variable immunodeficiency disorder: a casecontrol study versus sarcoidosis. Eur Respir J, 2013; 41(1): 115-22.

9. Chase, N.M., et al., Use of combination chemotherapy for treatment of granulomatous and lymphocytic interstitial lung disease (GLILD) in patients with common variable immunodeficiency (CVID). J Clin Immunol, 2013; 33(1): 30-9.

10. Mannina, A., et al., Clinical Predictors of a Diagnosis of Common Variable Immunodeficiency-related Granulomatous-Lymphocytic Interstitial Lung Disease. Ann Am Thorac Soc, 2016; 13(7): 1042-9.

11. Bates, C.A., et al., Granulomatous-lymphocytic lung disease shortens survival in common variable immunodeficiency. J Allergy Clin Immunol, 2004; 114(2): 415-21

12. Maglione, P.J., J.R. Overbey, and C. Cunningham-Rundles, Progression of Common Variable Immunodeficiency Interstitial Lung Disease Accompanies Distinct Pulmonary and Laboratory Findings. J Allergy Clin Immunol Pract, 2015;3(6): 941-50.

13. Rao, N., A.C. Mackinnon, and J.M. Routes, Granulomatous and lymphocytic interstitial lung disease: a spectrum of pulmonary histopathologic lesions in common variable immunodeficiency-histologic and immunohistochemical analyses of 16 cases. Hum Pathol, 2015; 46(9): 1306-14.

14. Park, J.E., et al., The HRCT appearances of granulomatous pulmonary disease in common variable immune deficiency. Eur J Radiol, 2005; 54(3): 359-64.

15. Jolles, S., et al., FDG PET-CT imaging of therapeutic response in granulomatous lymphocytic interstitial lung disease (GLILD) in common variable immunodeficiency (CVID). Clin Exp Immunol, 2017; 187(1): 138-145.

16. Rao, N., et al., Granulomatous and Lymphocytic Interstitial Lung Disease (GLILD) in Common Variable Immunodeficiency (CVID) - Histological and Immunohistochemical Analysis of 12 Cases of a Rare Entity. Modern Pathology, 2013; 26: 464a-464a.

17. Cereser, L., et al., Efficacy of rituximab as a single-agent therapy for the treatment of granulomatous and lymphocytic interstitial lung disease in patients with common variable immunodeficiency. J Allergy Clin Immunol Pract, 2019; 7(3): 1055-1057 e2. 
18. Beaton, T.J., et al., Granulomatous lymphocytic interstitial lung disease: limiting immunosuppressive therapy-a single-centre experience. Respirol Case Rep, 2020; 8(5): e00565.

\section{Tables}

Due to technical limitations, tables are only available as a download in the Supplemental Files section.

\section{Figures}

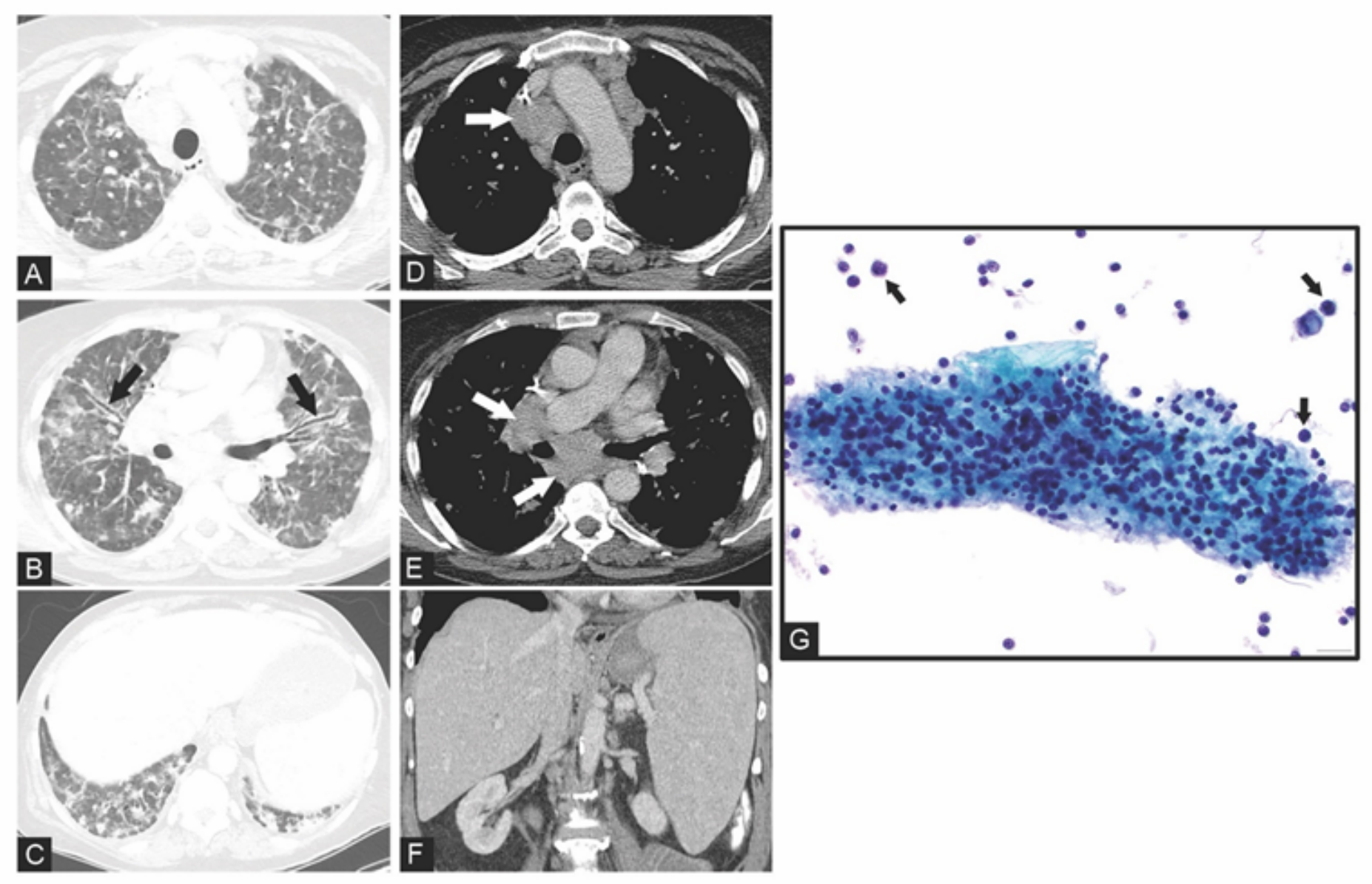

\section{Figure 1}

Patient 3 is a 66 year old female with CVID and GL-ILD: A, B and C. Three axial CT chest (lung windows) images demonstrate diffuse pulmonary nodules (part solid nodules, ground glass and solid nodules) with a perilymphatic and lower zone predominance. Additional patchy ground glass opacities are scattered throughout the lungs and there is smooth bronchial/bronchiolar wall thickening (black arrows). Infection was excluded via bronchoscopy with negative cultures. D, E and F. Two axial CT chest (soft tissue windows) images demonstrate enlarged mediastinal and bilateral hilar enlarged lymph nodes (white 
arrows); coronal chest CT (soft tissue windows) image demonstrates splenomegaly (16 cm in longest dimension) in the upper abdomen. G. Subcarinal lymph nodal aspirate demonstrates a subset of small to intermediate-sized lymphoid cells (black arrows; Papanicolaou stain, orginal magnification x400), confirmed by flow cytometric and immunophenotyping to represent low grade B cell lymphoma.

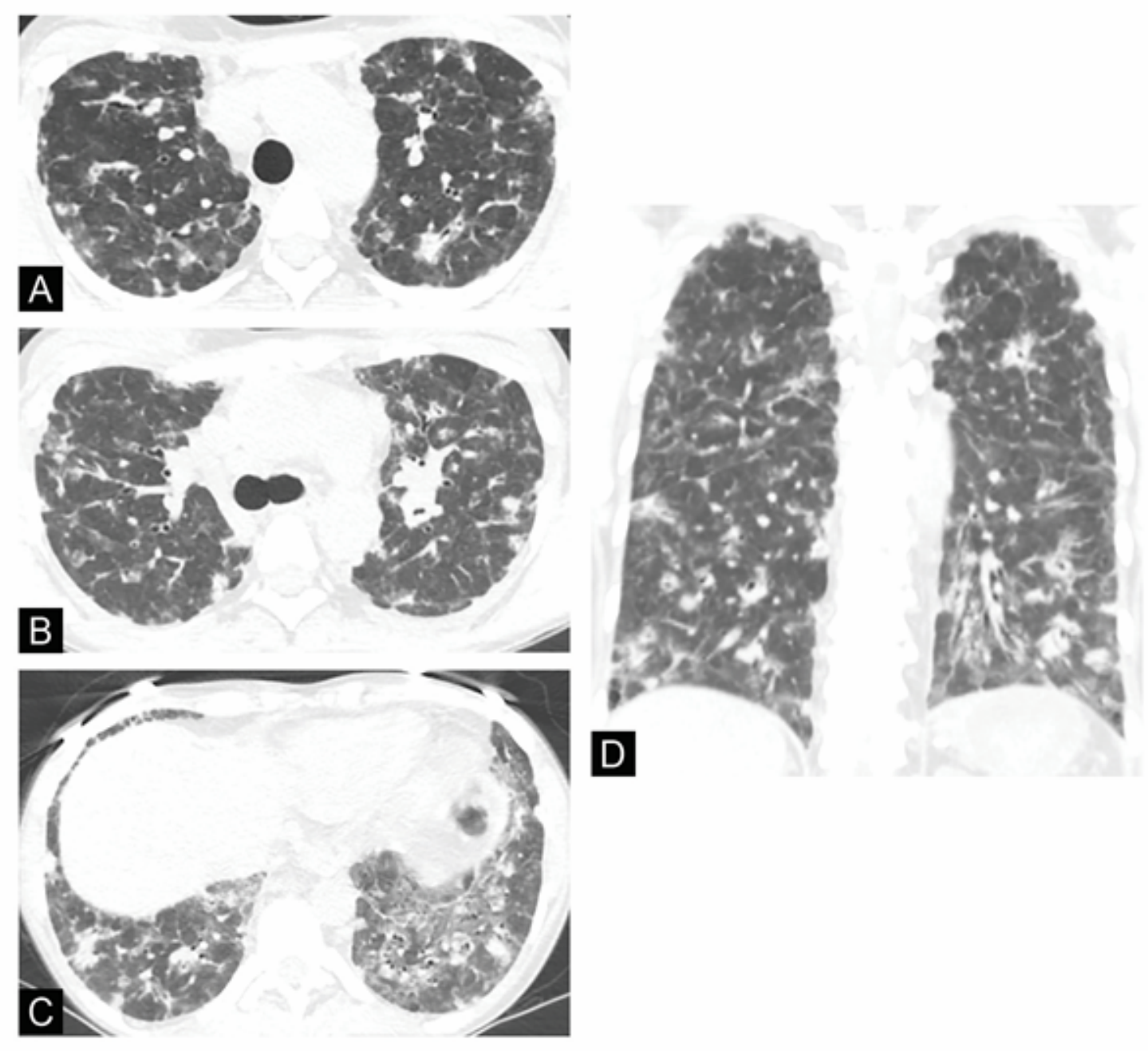

\section{Figure 2}

Patient 1 is a 36 year old female with CVID and GL-ILD: Three axial (A, B, and C) and one coronal (D) CT chest images (lung windows) demonstrate diffuse pulmonary nodules (mostly part solid nodules but also with ground glass and solid nodules) with an apical to basal gradient, most prominent in the lower lobes along with patchy ground glass opacities predominantly in the lower lobes. Infection was excluded via bronchoscopy with negative cultures and stains. 


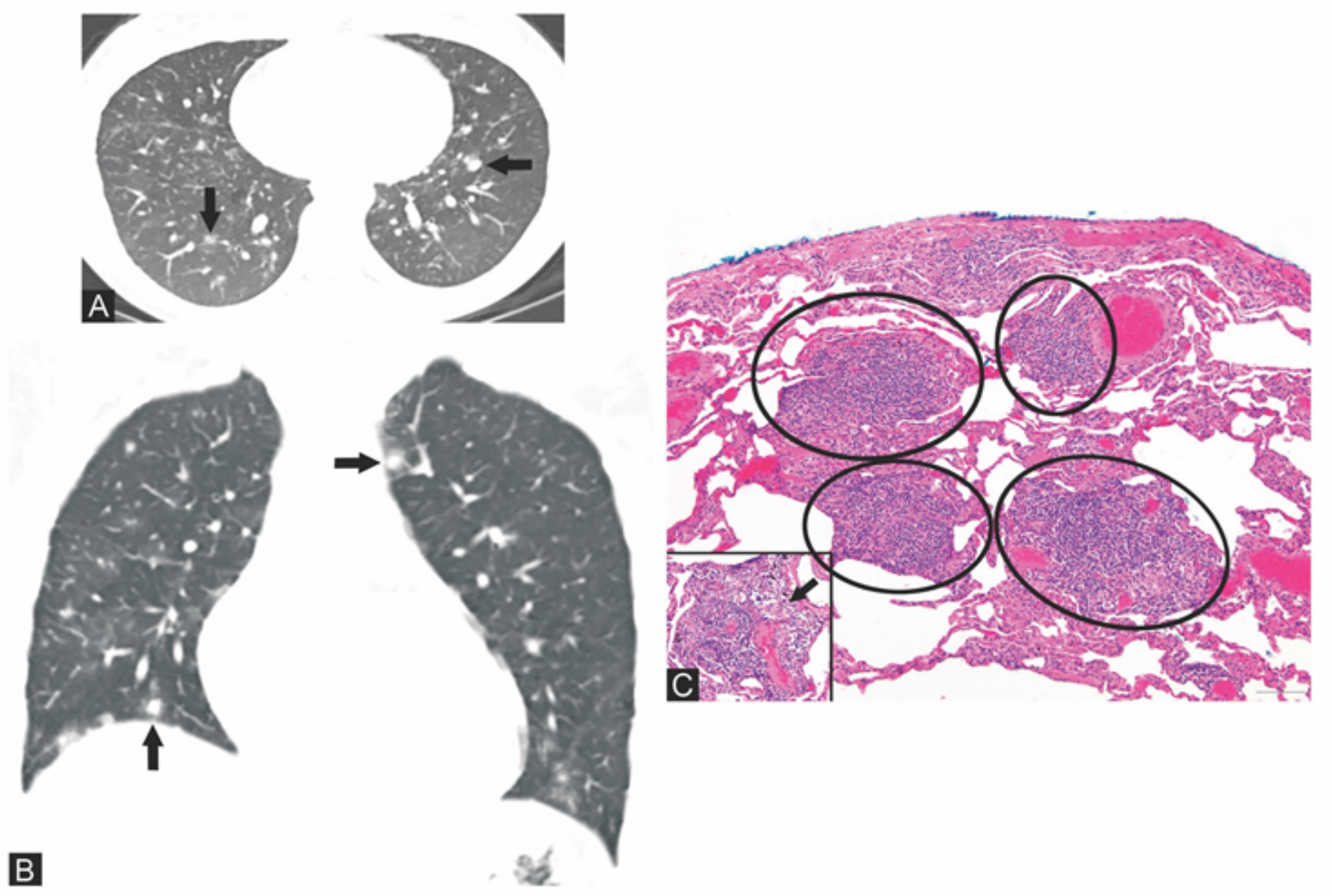

Figure 3

Patient 4 is a 30 year old female with CVID and mild GL-ILD. One coronal (A) CT chest (lung windows) images demonstrate a mild degree of scattered small pulmonary nodules (black arrows) (mostly part solid nodules but also with ground glass and solid nodules) with a lower zone predominance. Most of the nodules cluster along bronchovascular bundles, a perilymphatic distribution. B. Video-assisted thoracoscopic surgical wedge biopsies of the right upper, middle and lower lobes demonstrate multiple lymphoid aggregates diffusely throughout the lungs (black circles; hematoxylin \& esoin, original magnification $x 40$ ), follicular bronchiolitis and scatterred poorly-formed non-necrotizing granulomas (inset, black arrow; hematoxylin \& esoin, original magnification $x 200$ ). Infection was excluded with negative cultures and negative Grocott methenamine silver and AFB stains. 


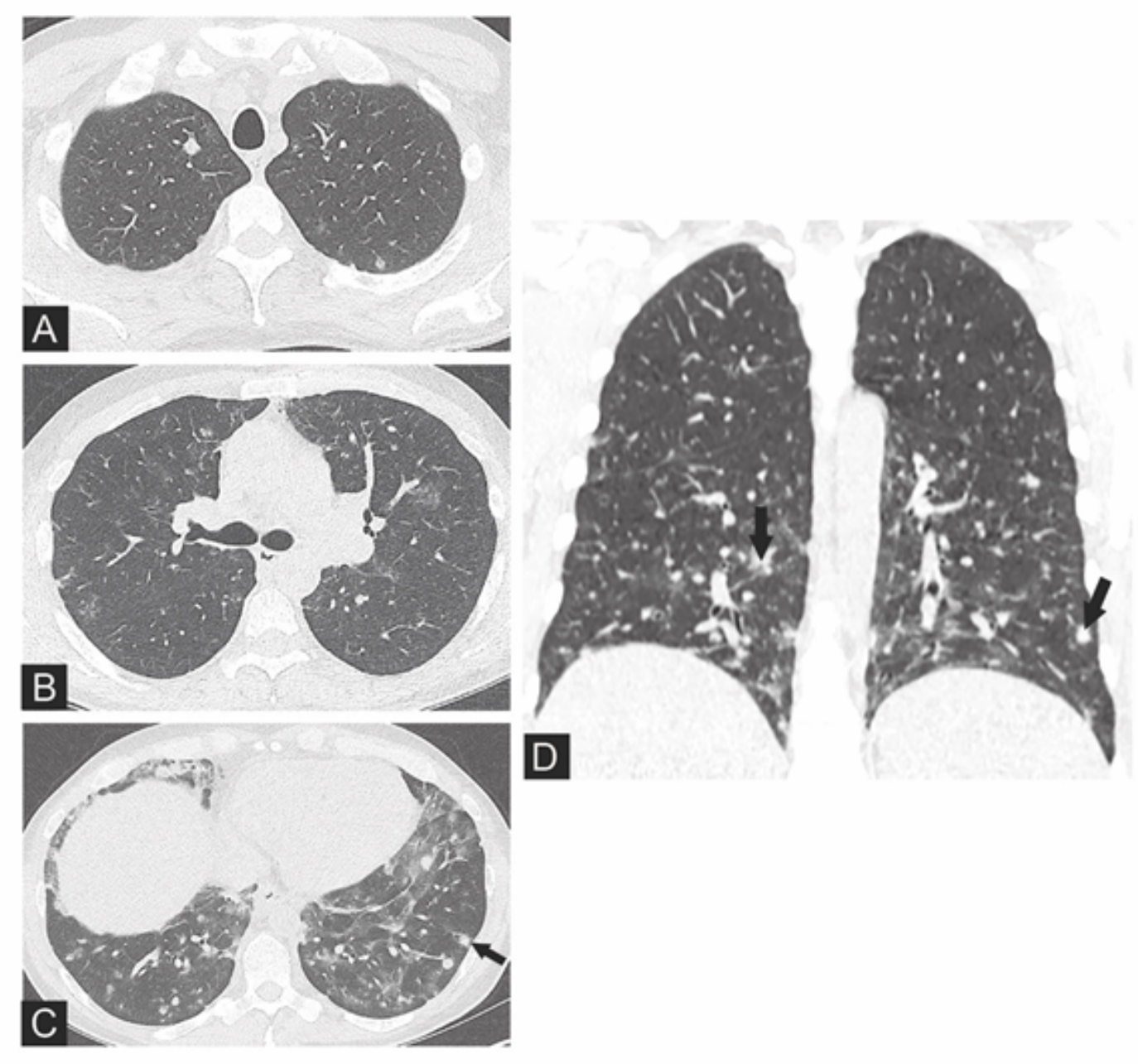

\section{Figure 4}

Patient 7 is a 50 year old male with CVID and a typical pattern of GL-ILD. Three axial and one coronal CT chest (lung windows) images demonstrate scattered bilateral pulmonary nodules bilaterally (ground glass, part solid and solid) (black arrows), most prominent in the lower lobes with patchy intermixed ground glass opacities. 

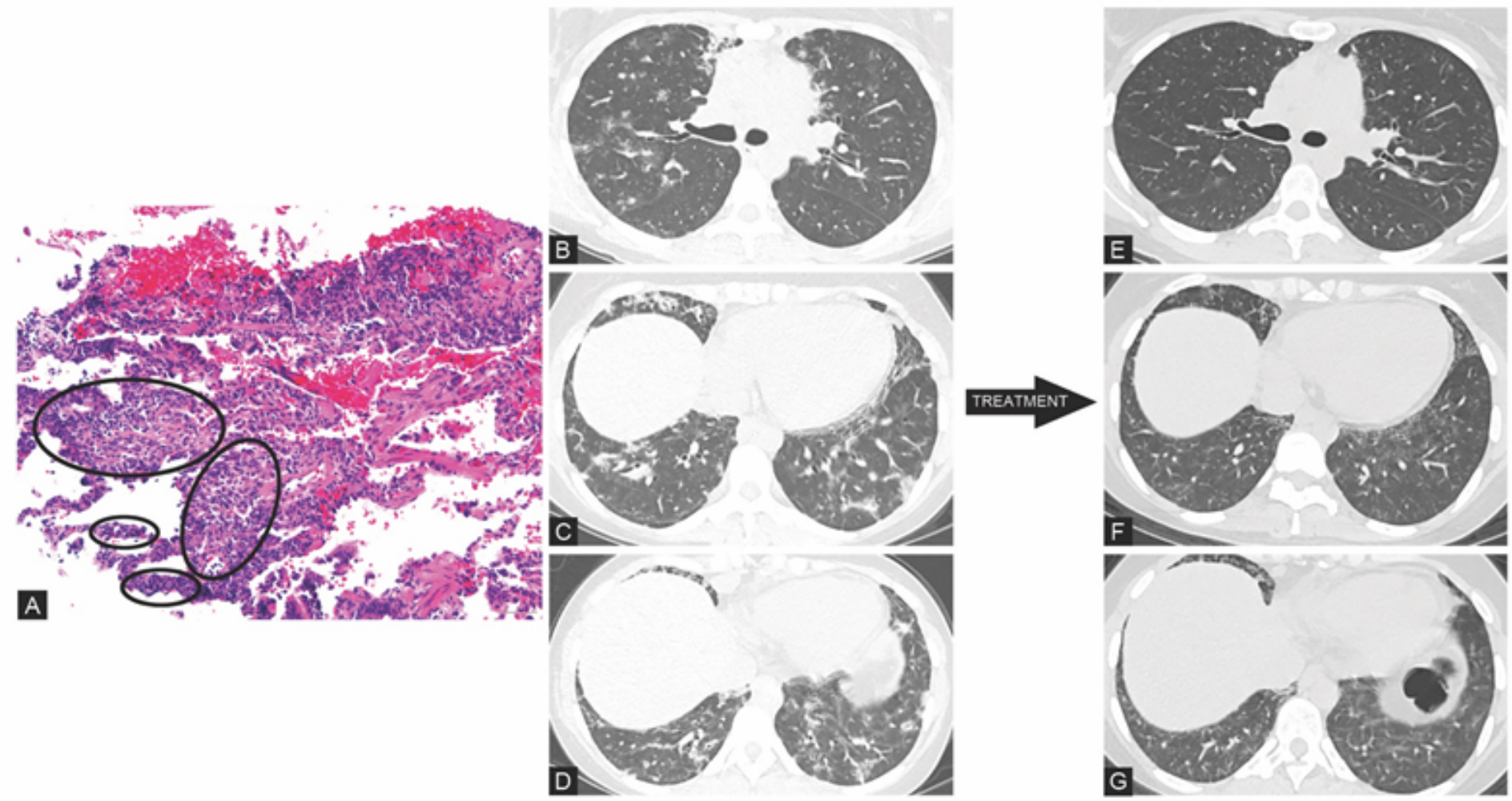

\section{Figure 5}

Patient 6 is a 33 year old female with CVID and GL-ILD. A. Bronchoscopic biopsy of the right middle lobe demonstrates prominent airway inflammation and organizing lung injury (not shown) with alveolar septal lymphocytic infiltrates (black circles; hematoxylin \& esoin, original magnification x 200); prominent airway inflammation and organizing lung injury was also seen (not shown). Infection was excluded with negative cultures and special stains. Gene arrangement studies by PCR did found no clonal lymphoid population. Pre-treatment March 2018: three axial CT chest (lung windows B, C and D) images demonstrate scattered bilateral pulmonary nodules bilaterally(ground glass, part solid and solid), with a lower zone predominance. Post-treatment with Sirolimus (with good response to autoimmune hemolytic anemia) October 2019: three axial CT chest (lung windows E, F and G) images demonstrates a treatment response with a significant reduction of the number and size of the pulmonary nodules.

\section{Supplementary Files}

This is a list of supplementary files associated with this preprint. Click to download. 
- Table1.docx

- Table2.docx

Page 15/15 